PROCEEDINGS OF THE

AMERICAN MATHEMATICAL SOCIETY

Volume 141, Number 8, August 2013, Pages 2729-2739

S 0002-9939(2013)11598-4

Article electronically published on April 5, 2013

\title{
A CHANGE OF SCALE FORMULA FOR A FUNCTION SPACE INTEGRAL ON $C_{a, b}[0, T]$
}

\author{
IL YOO, BONG JIN KIM, AND BYOUNG SOO KIM
}

(Communicated by Marius Junge)

\begin{abstract}
Cameron and Storvick discovered change of scale formulas for Wiener integrals of functionals in a Banach algebra $\mathcal{S}$ on classical Wiener space. Yoo and Skoug extended these results for functionals in the Fresnel class $\mathcal{F}(B)$ and in a generalized Fresnel class $\mathcal{F}_{A_{1}, A_{2}}$ on abstract Wiener space. We establish a relationship between a function space integral and a generalized analytic Feynman integral on $C_{a, b}[0, T]$ for functionals in a Banach algebra $\mathcal{S}\left(L_{a, b}^{2}[0, T]\right)$. Moreover, we obtain a change of scale formula for a function space integral on $C_{a, b}[0, T]$ of these functionals.
\end{abstract}

\section{INTRODUCTION}

It has long been known that Wiener measure and Wiener measurability behave badly under the change of scale transformation 2 and under translations [1. Cameron and Storvick [5] expressed the analytic Feynman integral on classical Wiener space as a limit of Wiener integrals. In doing so, they discovered nice change of scale formulas for Wiener integrals on classical Wiener space $\left(C_{0}[0,1], m_{w}\right)$ 4. In [12, 13, Yoo and Skoug extended these results to an abstract Wiener space $(H, B, \nu)$. Moreover, Yoo, Song, Kim and Chang [14, 15] established a change of scale formula for Wiener integrals of some unbounded functionals on (a product) abstract Wiener space.

On the other hand, Chang, Choi, Lee and Skoug [6, 7, 8, 9] studied a generalized analytic Feynman integral on a very general function space $C_{a, b}[0, T]$ rather than the Wiener space $C_{0}[0, T]$. The Wiener process $W$ on $C_{0}[0, T] \times[0, T]$ is free of drift and is stationary in time, while the stochastic process $Y$ on $C_{a, b}[0, T] \times[0, T]$ is subject to a drift $a(t)$ and is nonstationary in time. For more details, see [6, 9 .

In this paper, we establish a relationship between a function space integral and a generalized analytic Feynman integral on $C_{a, b}[0, T]$ for functionals in a Banach algebra $\mathcal{S}\left(L_{a, b}^{2}[0, T]\right)$. That is, we express a generalized analytic Feynman integral on $C_{a, b}[0, T]$ as a limit of function space integrals on $C_{a, b}[0, T]$. Moreover, we obtain a change of scale formula for a function space integral on $C_{a, b}[0, T]$ of these functionals.

Received by the editors January 4, 2011 and, in revised form, October 28, 2011.

2000 Mathematics Subject Classification. Primary 28C20; Secondary 60J25, 60J65.

Key words and phrases. Change of scale formula, function space integral, generalized analytic Feynman integral.

This research was supported by the Basic Science Research Program through the National Research Foundation of Korea (NRF) funded by the Ministry of Education, Science and Technology (2010-0022563). 
By choosing $a(t)=0$ and $b(t)=t$ on $[0, T]$, the function space $C_{a, b}[0, T]$ reduces to the Wiener space $C_{0}[0, T]$, and so the results in [4, 5] are immediate corollaries of the results in this paper.

\section{Definitions AND PRELIMINARIES}

Let $a(t)$ be an absolutely continuous real valued function on $[0, T]$ with $a(0)=0$, $a^{\prime}(t) \in L^{2}[0, T]$, and $b(t)$ be a strictly increasing continuous differentiable real valued function with $b(0)=0$. The generalized Brownian motion process $Y$ determined by $a(\cdot)$ and $b(\cdot)$ is a Gaussian process with mean function $a(t)$ and covariance function $r(s, t)=\min \{b(s), b(t)\}$. For more detail, see Section 2 of [6]. By Theorem 14.2 in [11, the probability measure $\mu$ induced by $Y$, taking a separable version, is supported by $C_{a, b}[0, T]$ (which is equivalent to the Banach space of continuous functions $x$ on $[0, T]$ with $x(0)=0$ under the sup norm). Let $W\left(C_{a, b}[0, T]\right)$ denote the class of all $\mu$ measurable subsets of $C_{a, b}[0, T]$.

A subset $E$ of $C_{a, b}[0, T]$ is said to be scale-invariant measurable provided $\rho E$ is in $W\left(C_{a, b}[0, T]\right)$ for every $\rho>0$, and a scale-invariant measurable set $N$ is said to be scale-invariant null provided $\mu(\rho N)=0$ for every $\rho>0$. A property that holds except on a scale-invariant null set is said to hold scale-invariant almost everywhere (s-a.e.) [10].

Let $L_{a, b}^{2}[0, T]$ be the Hilbert space of continuous functions on $[0, T]$ which are Lebesgue measurable and square integrable with respect to the Lebesgue Stieltjes measures on $[0, T]$ induced by $a(\cdot)$ and $b(\cdot)$; i.e.,

$$
L_{a, b}^{2}[0, T]=\left\{v: \int_{0}^{T} v^{2}(t) d b(t)<\infty \text { and } \int_{0}^{T} v^{2}(t) d|a|(t)<\infty\right\},
$$

where $|a|(t)$ denotes the total variation of the function $a$ on the interval $[0, t]$.

For $u, v \in L_{a, b}^{2}[0, T]$, let

$$
(u, v)_{a, b}=\int_{0}^{T} u(t) v(t) d[b(t)+|a|(t)] .
$$

Then $(\cdot, \cdot)_{a, b}$ is an inner product on $L_{a, b}^{2}[0, T]$ and $\|u\|_{a, b}=\sqrt{(u, u)_{a, b}}$ is a norm on $L_{a, b}^{2}[0, T]$. Furthermore, $\left(L_{a, b}^{2}[0, T],\|\cdot\|_{a, b}\right)$ is a separable Hilbert space.

Let $\left\{\phi_{j}\right\}$ be a complete orthonormal set of real valued functions of bounded variation on $[0, T]$ such that

$$
\left(\phi_{j}, \phi_{k}\right)_{a, b}= \begin{cases}0, & j \neq k \\ 1, & j=k\end{cases}
$$

and for each $v \in L_{a, b}^{2}[0, T]$, let

$$
v_{n}(t)=\sum_{j=1}^{n}\left(v, \phi_{j}\right)_{a, b} \phi_{j}(t)
$$

for $n=1,2, \ldots$ Then for each $v \in L_{a, b}^{2}[0, T]$, the Paley-Wiener-Zygmund stochastic integral $\langle v, x\rangle$ is defined by the formula

$$
\langle v, x\rangle=\lim _{n \rightarrow \infty} \int_{0}^{T} v_{n}(t) d x(t)
$$

for all $x \in C_{a, b}[0, T]$ for which the limit exists. 
We denote the function space integral of a $W\left(C_{a, b}[0, T]\right)$-measurable functional $F$ by

$$
\int_{C_{a, b}[0, T]} F(x) d \mu(x)
$$

whenever the integral exists.

Let $\mathbb{C}_{+}$and $\mathbb{C}_{+}^{\sim}$ denote the set of complex numbers with positive real part and complex numbers with nonnegative real part, respectively.

Definition 2.1. Let $F$ be a complex valued measurable functional on $C_{a, b}[0, T]$ such that the function space integral

$$
J_{F}(\lambda)=\int_{C_{a, b}[0, T]} F\left(\lambda^{-1 / 2} x\right) d \mu(x)
$$

exists as a finite number for all $\lambda>0$. If there exists a function $J_{F}^{*}(\lambda)$ analytic in $\mathbb{C}_{+}$such that $J_{F}^{*}(\lambda)=J_{F}(\lambda)$ for all $\lambda>0$, then $J_{F}^{*}(\lambda)$ is defined to be the analytic function space integral of $F$ over $C_{a, b}[0, T]$ with parameter $\lambda$, and for $\lambda \in \mathbb{C}_{+}$we write

$$
\int_{C_{a, b}[0, T]}^{\mathrm{an}_{\lambda}} F(x) d \mu(x)=J_{F}^{*}(\lambda)
$$

If the following limit exists for nonzero real $q$, then we call it the generalized analytic Feynman integral of $F$ over $C_{a, b}[0, T]$ with parameter $q$ and we write

$$
\int_{C_{a, b}[0, T]}^{\operatorname{anf}_{q}} F(x) d \mu(x)=\lim _{\lambda \rightarrow-i q} \int_{C_{a, b}[0, T]}^{\operatorname{an}_{\lambda}} F(x) d \mu(x),
$$

where $\lambda$ approaches $-i q$ through $\mathbb{C}_{+}$.

Definition 2.2. Let $M\left(L_{a, b}^{2}[0, T]\right)$ denote the space of complex valued countably additive Borel measures on $L_{a, b}^{2}[0, T]$. The Banach algebra $\mathcal{S}\left(L_{a, b}^{2}[0, T]\right)$ is the space of all $s$-equivalence classes of functionals $F$ on $C_{a, b}[0, T]$ which have the form

$$
F(x)=\int_{L_{a, b}^{2}[0, T]} \exp \{i\langle v, x\rangle\} d f(v),
$$

where the associated measure $f$ is an element of $M\left(L_{a, b}^{2}[0, T]\right)$.

Remark 2.3. Let $a(t)=0$ and $b(t)=t$ on $[0, T]$. Then:

(i) The analytic function space integral $\int_{C_{a, b}[0, T]}^{a_{\lambda}} F(x) d \mu(x)$ reduces to the analytic Wiener integral $\int_{C_{0}[0, T]}^{a_{1} w_{\lambda}} F(x) d m_{w}(x)$.

(ii) The generalized analytic Feynman integral $\int_{C_{a, b}[0, T]}^{\operatorname{anf}_{q}} F(x) d \mu(x)$ reduces to the analytic Feynman integral $\int_{C_{0}[0, T]}^{\operatorname{anf}_{q}} F(x) d m_{w}(x)$.

(iii) The Banach algebra $\mathcal{S}\left(L_{a, b}^{2}[0, T]\right)$ reduces to the Banach algebra $\mathcal{S}$ introduced by Cameron and Storvick in [3].

The following notation is used throughout this paper:

and

$$
\left(u, a^{\prime}\right)=\int_{0}^{T} u(t) a^{\prime}(t) d t=\int_{0}^{T} u(t) d a(t)
$$

$$
\left(u^{2}, b^{\prime}\right)=\int_{0}^{T} u^{2}(t) b^{\prime}(t) d t=\int_{0}^{T} u^{2}(t) d b(t)
$$


for $u \in L_{a, b}^{2}[0, T]$. Furthermore, for all $\lambda \in \mathbb{C}_{+}^{\sim}, \sqrt{\lambda}$ is always chosen to have positive real part.

Now we state a very fundamental integration formula on the function space $C_{a, b}[0, T]$. It is a generalization of the Wiener integration formula on Wiener space.

Let $\left\{\phi_{1}, \ldots, \phi_{n}\right\}$ be an orthonormal set in $L_{a, b}^{2}[0, T]$ and, for $k=1, \ldots, n$, let

$$
A_{k} \equiv\left(\phi_{k}, a^{\prime}\right)=\int_{0}^{T} \phi_{k}(t) d a(t)
$$

and

$$
B_{k} \equiv\left(\phi_{k}^{2}, b^{\prime}\right)=\int_{0}^{T} \phi_{k}^{2}(t) d b(t) .
$$

Let $f: \mathbb{R}^{n} \rightarrow \mathbb{R}$ be a Lebesgue measurable function. Then

$$
\begin{aligned}
& \int_{C_{a, b}[0, T]} f\left(\left\langle\phi_{1}, x\right\rangle, \ldots,\left\langle\phi_{n}, x\right\rangle\right) d \mu(x) \\
& =\left(\prod_{k=1}^{n} 2 \pi B_{k}\right)^{-1 / 2} \int_{\mathbb{R}^{n}} f\left(u_{1}, \ldots, u_{n}\right) \exp \left\{-\sum_{k=1}^{n} \frac{\left(u_{k}-A_{k}\right)^{2}}{2 B_{k}}\right\} d u_{1} \cdots d u_{n}
\end{aligned}
$$

in the sense that if either side exists, both sides exist and equality holds.

We finish this section by introducing the existence theorem (Remark 4.2 of [9]) of the analytic function space integral and the generalized analytic Feynman integral on $C_{a, b}[0, T]$ for functionals in $\mathcal{S}\left(L_{a, b}^{2}[0, T]\right)$.

Theorem 2.4. Let $\lambda \in \mathbb{C}_{+}$. Let $F \in \mathcal{S}\left(L_{a, b}^{2}[0, T]\right)$ be given by (2.4) and suppose that the associated measure $f$ satisfies the condition

$$
\int_{L_{a, b}^{2}[0, T]} \exp \left\{-\beta \int_{0}^{T}|v(t)| d|a|(t)\right\}|d f(v)|<\infty,
$$

where $\beta=\operatorname{Im}\left(\lambda^{-1 / 2}\right)$. Then the analytic function space integral of $F$ exists and is given by

$$
\int_{C_{a, b}[0, T]}^{\mathrm{an}_{\lambda}} F(x) d \mu(x)=\int_{L_{a, b}^{2}[0, T]} \exp \left\{-\frac{1}{2 \lambda}\left(v^{2}, b^{\prime}\right)+i \lambda^{-1 / 2}\left(v, a^{\prime}\right)\right\} d f(v) .
$$

Furthermore, let $q$ be a nonzero real number and suppose that the associated measure $f$ satisfies the condition

$$
\int_{L_{a, b}^{2}[0, T]} \exp \left\{|2 q|^{-1 / 2} \int_{0}^{T}|v(t)| d|a|(t)\right\}|d f(v)|<\infty .
$$

Then the generalized analytic Feynman integral of $F$ exists and is given by

$$
\int_{C_{a, b}[0, T]}^{\operatorname{anf}_{\mathrm{q}}} F(x) d \mu(x)=\int_{L_{a, b}^{2}[0, T]} \exp \left\{-\frac{i}{2 q}\left(v^{2}, b^{\prime}\right)+i\left(\frac{i}{q}\right)^{1 / 2}\left(v, a^{\prime}\right)\right\} d f(v) .
$$




\section{A ChANGe OF SCALE FORMUla}

FOR A FUNCTION SPACE INTEGRAL ON $C_{a, b}[0, T]$

We begin this section by giving a function space integration formula that is useful in the proof of Theorem 3.2.

Lemma 3.1. Let $\lambda \in \mathbb{C}_{+}$, let $\left\{\phi_{1}, \ldots, \phi_{n}\right\}$ be an orthonormal set in $L_{a, b}^{2}[0, T]$ and let $v \in L_{a, b}^{2}[0, T]$. Then if

$$
K \equiv \int_{C_{a, b}[0, T]} \exp \left\{\frac{1-\lambda}{2} \sum_{k=1}^{n} \frac{\left\langle\phi_{k}, x\right\rangle^{2}}{B_{k}}+\left(\lambda^{1 / 2}-1\right) \sum_{k=1}^{n} \frac{A_{k}\left\langle\phi_{k}, x\right\rangle}{B_{k}}+i\langle v, x\rangle\right\} d \mu(x),
$$

it follows that

$$
\begin{aligned}
K= & \lambda^{-n / 2} \exp \left\{-\frac{1}{2}\left(\psi_{n}^{2}, b^{\prime}\right)+i\left(\psi_{n}, a^{\prime}\right)\right. \\
& \left.+\sum_{k=1}^{n}\left[-\frac{1}{2 \lambda} B_{k}\left(\phi_{k}, v\right)_{a, b}^{2}+i \lambda^{-1 / 2} A_{k}\left(\phi_{k}, v\right)_{a, b}\right]\right\},
\end{aligned}
$$

where

$$
\psi_{n}=v-\sum_{k=1}^{n}\left(\phi_{k}, v\right)_{a, b} \phi_{k} .
$$

Proof. By the Gram-Schmidt process, we obtain $\phi_{n+1} \in L_{a, b}^{2}[0, T]$ such that $\left\{\phi_{1}, \ldots\right.$, $\left.\phi_{n}, \phi_{n+1}\right\}$ is an orthonormal set in $L_{a, b}^{2}[0, T]$ and

$$
v=\sum_{k=1}^{n+1} c_{k} \phi_{k}
$$

where

$$
c_{k}= \begin{cases}\left(\phi_{k}, v\right)_{a, b}, & k=1, \ldots, n, \\ {\left[\|v\|_{a, b}^{2}-\sum_{j=1}^{n}\left(\phi_{j}, v\right)_{a, b}^{2}\right]^{1 / 2},} & k=n+1 .\end{cases}
$$

In fact $\psi_{n}=\left\|\psi_{n}\right\|_{a, b} \phi_{n+1}=c_{n+1} \phi_{n+1}$, where $\psi_{n}$ is given by (3.3). Hence

$$
\begin{aligned}
K= & \int_{C_{a, b}[0, T]} \exp \left\{\frac{1-\lambda}{2} \sum_{k=1}^{n} \frac{\left\langle\phi_{k}, x\right\rangle^{2}}{B_{k}}+\left(\lambda^{1 / 2}-1\right) \sum_{k=1}^{n} \frac{A_{k}\left\langle\phi_{k}, x\right\rangle}{B_{k}}\right. \\
& \left.+i \sum_{k=1}^{n+1} c_{k}\left\langle\phi_{k}, x\right\rangle\right\} d \mu(x) .
\end{aligned}
$$

By the integration formula (2.7), we have

$$
\begin{aligned}
K= & \left(\prod_{k=1}^{n+1} 2 \pi B_{k}\right)^{-1 / 2} \int_{\mathbb{R}^{n+1}} \exp \left\{\frac{1-\lambda}{2} \sum_{k=1}^{n} \frac{u_{k}^{2}}{B_{k}}+\left(\lambda^{1 / 2}-1\right) \sum_{k=1}^{n} \frac{A_{k} u_{k}}{B_{k}}\right. \\
& \left.+i \sum_{k=1}^{n+1} c_{k} u_{k}-\sum_{k=1}^{n+1} \frac{\left(u_{k}-A_{k}\right)^{2}}{2 B_{k}}\right\} d u_{1} \cdots d u_{n+1} .
\end{aligned}
$$

Now we evaluate the above integral using the integration formula

$$
\int_{\mathbb{R}} \exp \left\{-\alpha u^{2}+i \beta u\right\} d u=\left(\frac{\pi}{\alpha}\right)^{1 / 2} \exp \left\{-\frac{\beta^{2}}{4 \alpha}\right\}, \quad \alpha \in \mathbb{C}_{+},
$$


to obtain

$$
K=\lambda^{-n / 2} \exp \left\{-\frac{B_{n+1}}{2} c_{n+1}^{2}+i A_{n+1} c_{n+1}+\sum_{k=1}^{n}\left[-\frac{1}{2 \lambda} B_{k} c_{k}^{2}+i \lambda^{-1 / 2} A_{k} c_{k}\right]\right\} .
$$

Finally, since

$$
B_{n+1} c_{n+1}^{2}=\left(\phi_{n+1}^{2}, b^{\prime}\right) c_{n+1}^{2}=\left(\phi_{n+1}^{2} c_{n+1}^{2}, b^{\prime}\right)=\left(\psi_{n}^{2}, b^{\prime}\right)
$$

and

we have (3.2).

$$
A_{n+1} c_{n+1}=\left(\phi_{n+1}, a^{\prime}\right) c_{n+1}=\left(\psi_{n}, a^{\prime}\right)
$$

Now we give a relationship between the function space integral and the generalized analytic Feynman integral on $C_{a, b}[0, T]$ for functionals in $\mathcal{S}\left(L_{a, b}^{2}[0, T]\right)$. But we need a further assumption on $a(t)$ to obtain the relationship.

Theorem 3.2. Let $|a(t)|=c b(t)$ on $[0, T]$ for some constant $c \geq 0$. Let $\left\{\phi_{n}\right\}$ be a complete orthonormal set of functionals in $L_{a, b}^{2}[0, T]$. Let $q$ be a nonzero real number and let $\left\{\lambda_{n}\right\}$ be a sequence of complex numbers in $\mathbb{C}_{+}$such that $\lambda_{n} \rightarrow-i q$. Let $F \in \mathcal{S}\left(L_{a, b}^{2}[0, T]\right)$ be given by (2.4) and suppose that the associated measure $f$ satisfies condition (2.10). Then we have

$$
\begin{aligned}
\int_{C_{a, b}[0, T]}^{\operatorname{anf}_{\mathrm{q}}} F(x) d \mu(x)=\lim _{n \rightarrow \infty} \lambda_{n}^{n / 2} \int_{C_{a, b}[0, T]} \exp \left\{\frac{1-\lambda_{n}}{2} \sum_{k=1}^{n} \frac{\left\langle\phi_{k}, x\right\rangle^{2}}{B_{k}}\right. \\
\left.+\left(\lambda_{n}^{1 / 2}-1\right) \sum_{k=1}^{n} \frac{A_{k}\left\langle\phi_{k}, x\right\rangle}{B_{k}}\right\} F(x) d \mu(x) .
\end{aligned}
$$

Proof. Let $\Gamma\left(\lambda_{n}\right)$ be the function space integral on the right hand side of (3.4). Then by (2.4) and the Fubini theorem,

$$
\Gamma\left(\lambda_{n}\right)=\int_{L_{a, b}^{2}[0, T]} K\left(v, \lambda_{n}\right) d f(v)
$$

where

$$
\begin{aligned}
K\left(v, \lambda_{n}\right)= & \int_{C_{a, b}[0, T]} \exp \left\{\frac{1-\lambda_{n}}{2} \sum_{k=1}^{n} \frac{\left\langle\phi_{k}, x\right\rangle^{2}}{B_{k}}\right. \\
& \left.+\left(\lambda_{n}^{1 / 2}-1\right) \sum_{k=1}^{n} \frac{A_{k}\left\langle\phi_{k}, x\right\rangle}{B_{k}}+i\langle v, x\rangle\right\} d \mu(x) .
\end{aligned}
$$

By Lemma 3.1,

$$
\begin{aligned}
K\left(v, \lambda_{n}\right)= & \lambda_{n}^{-n / 2} \exp \left\{-\frac{1}{2}\left(\psi_{n}^{2}, b^{\prime}\right)+i\left(\psi_{n}, a^{\prime}\right)\right. \\
& \left.+\sum_{k=1}^{n}\left[-\frac{1}{2 \lambda_{n}} B_{k}\left(\phi_{k}, v\right)_{a, b}^{2}+i \lambda_{n}^{-1 / 2} A_{k}\left(\phi_{k}, v\right)_{a, b}\right]\right\},
\end{aligned}
$$

where $\psi_{n}$ is given as in (3.3). Now we examine each term in the exponential on the right hand side of the above expression. Since $\psi_{n} \rightarrow 0$ in $L_{a, b}^{2}[0, T]$ as $n \rightarrow \infty$,

$$
\left|\left(\psi_{n}^{2}, b^{\prime}\right)\right|=\left|\int_{0}^{T}\left(\psi_{n}(t)\right)^{2} d b(t)\right| \leq\left\|\psi_{n}\right\|_{a, b}^{2} \rightarrow 0
$$


as $n \rightarrow \infty$. Furthermore, by the Hölder inequality,

$$
\left|\left(\psi_{n}, a^{\prime}\right)\right|=\left|\int_{0}^{T} \psi_{n}(t) d a(t)\right| \leq\left\|\psi_{n}\right\|_{a, b}\|a\|^{1 / 2} \rightarrow 0
$$

as $n \rightarrow \infty$, where $\|a\|$ denotes the total variation of $a$ on $[0, T]$. Hence we know that the first two terms in the exponential of (3.5) go to 0 as $n \rightarrow \infty$. Moreover,

$$
\left|\sum_{k=1}^{n} A_{k}\left(\phi_{k}, v\right)_{a, b}-\left(v, a^{\prime}\right)\right|=\left|\left(\sum_{k=1}^{n}\left(\phi_{k}, v\right)_{a, b} \phi_{k}-v, a^{\prime}\right)\right|=\left|\left(\psi_{n}, a^{\prime}\right)\right| \rightarrow 0
$$

as above, and so

$$
\sum_{k=1}^{n} A_{k}\left(\phi_{k}, v\right)_{a, b} \rightarrow\left(v, a^{\prime}\right)
$$

as $n \rightarrow \infty$. Now it remains to consider the third term in the exponential of (3.5). Since

$$
0=\left(\phi_{j}, \phi_{k}\right)_{a, b}=(1+c) \int_{0}^{T} \phi_{j}(t) \phi_{k}(t) d b(t)
$$

for all $j \neq k$, we have

$$
\int_{0}^{T} \phi_{j}(t) \phi_{k}(t) d b(t)=0
$$

for all $j \neq k$, and so

$$
\sum_{k=1}^{n} B_{k}\left(\phi_{k}, v\right)_{a, b}^{2}=\left(\psi_{n}^{2}, b^{\prime}\right)+2 \sum_{k=1}^{n}\left(\phi_{k}, v\right)_{a, b}\left(\phi_{k} v, b^{\prime}\right)-\left(v^{2}, b^{\prime}\right) .
$$

But

and by the Hölder inequality

$$
\left|\left(\psi_{n}^{2}, b^{\prime}\right)\right| \leq\left\|\psi_{n}\right\|_{a, b}^{2} \rightarrow 0
$$

$$
\begin{aligned}
& \left|\sum_{k=1}^{n}\left(\phi_{k}, v\right)_{a, b}\left(\phi_{k} v, b^{\prime}\right)-\left(v^{2}, b^{\prime}\right)\right|=\left|\left(\psi_{n} v, b^{\prime}\right)\right| \\
& \leq\left(\int_{0}^{T}\left(\psi_{n}(t)\right)^{2} d b(t)\right)^{1 / 2}\left(\int_{0}^{T}(v(t))^{2} d b(t)\right)^{1 / 2} \leq\left\|\psi_{n}\right\|_{a, b}\|v\|_{a, b} \rightarrow 0
\end{aligned}
$$

as $n \rightarrow \infty$. Hence from (3.9) we have

$$
\sum_{k=1}^{n} B_{k}\left(\phi_{k}, v\right)_{a, b}^{2} \rightarrow\left(v^{2}, b^{\prime}\right)
$$

as $n \rightarrow \infty$.

From the four convergences in (3.6), (3.7), (3.8) and (3.10), we see that

$$
\lim _{n \rightarrow \infty} \lambda_{n}^{n / 2} K\left(v, \lambda_{n}\right)=\exp \left\{-\frac{i}{2 q}\left(v^{2}, b^{\prime}\right)+i\left(\frac{i}{q}\right)^{1 / 2}\left(v, a^{\prime}\right)\right\} .
$$

Since $f$ satisfies condition (2.10) we apply the dominated convergence theorem to obtain

$$
\begin{aligned}
\lim _{n \rightarrow \infty} \lambda_{n}^{n / 2} \Gamma\left(\lambda_{n}\right) & =\lim _{n \rightarrow \infty} \lambda_{n}^{n / 2} \int_{L_{a, b}^{2}[0, T]} K\left(v, \lambda_{n}\right) d f(v) \\
& =\int_{L_{a, b}^{2}[0, T]} \exp \left\{-\frac{i}{2 q}\left(v^{2}, b^{\prime}\right)+i\left(\frac{i}{q}\right)^{1 / 2}\left(v, a^{\prime}\right)\right\} d f(v) .
\end{aligned}
$$


By (2.11) in Theorem 2.4, the proof is completed.

Let $a(t)=0$ and $b(t)=t$ on $[0, T]$. Then $a(t)$ satisfies the assumption in Theorem 3.2, condition (2.10) is satisfied for any measure $f$ in $M\left(L_{a, b}^{2}[0, T]\right)$ and

$$
A_{k}=\left(\phi_{k}, 0\right)=0, \quad B_{k}=\left(\phi_{k}^{2}, 1\right)=\left(\phi_{k}^{2}, 1\right)_{a, b}=1 .
$$

Moreover, as we see in Remark 2.3 above, the generalized analytic Feynman integral $\int_{C_{a, b}[0, T]}^{\operatorname{anf}_{q}} F(x) d \mu(x)$ on the function space $C_{a, b}[0, T]$ reduces to the analytic Feynman integral $\int_{C_{0}[0, T]}^{\operatorname{anf}_{q}} F(x) d m_{w}(x)$ on Wiener space $C_{0}[0, T]$. Hence we have the following relationship between the Wiener integral and the analytic Feynman integral on $C_{0}[0, T]$ for functionals in a Banach algebra $\mathcal{S}$.

Corollary 3.3 (Theorem 2 of [5]). Let $\left\{\phi_{n}\right\}$ be a complete orthonormal set of functionals in $L^{2}[0, T]$. Let $q$ be a nonzero real number and let $\left\{\lambda_{n}\right\}$ be a sequence of complex numbers in $\mathbb{C}_{+}$such that $\lambda_{n} \rightarrow-i q$. Let $F$ be a functional in a Banach algebra $\mathcal{S}$; that is,

$$
F(x)=\int_{L^{2}[0, T]} \exp \{i\langle v, x\rangle\} d f(v),
$$

where $f$ is a complex Borel measure on $L^{2}[0, T]$. Then

$$
\int_{C_{0}[0, T]}^{\text {anf }_{\mathrm{q}}} F(x) d m_{w}(x)=\lim _{n \rightarrow \infty} \lambda_{n}^{n / 2} \int_{C_{0}[0, T]} \exp \left\{\frac{1-\lambda_{n}}{2} \sum_{k=1}^{n}\left\langle\phi_{k}, x\right\rangle^{2}\right\} F(x) d m_{w}(x) .
$$

In our next example we give $a(t), b(t)$ and a functional $F$ in $\mathcal{S}\left(L_{a, b}^{2}[0, T]\right)$ to show that the relationship (3.4) is not necessarily valid if $a(t)$ does not satisfy the assumption in Theorem 3.2. For notational and computational convenience let $T=1$.

Example 3.4. Let $a(t)=t^{2} / 2$ and $b(t)=t$ for $0 \leq t \leq 1$ and let $q$ be a nonzero real number. Note that $a(t)$ does not satisfy the assumption in Theorem 3.2. Let $\phi_{1}(t)=1 / \sqrt{1+t}$ for $0 \leq t \leq 1$. Then

$$
\left\|\phi_{1}\right\|_{a, b}=\int_{0}^{1} \frac{1}{1+t} d[b(t)+|a|(t)]=\int_{0}^{1} \frac{1}{1+t}(1+t) d t=1 .
$$

Of course we can choose $\phi_{2}, \phi_{3}, \ldots$ in $L_{a, b}^{2}[0,1]$ such that $\left\{\phi_{n}\right\}$ is a complete orthonormal set in $L_{a, b}^{2}[0,1]$. Let $f$ be the Dirac measure concentrated at $v \equiv 1$ and let $F$ be given by (2.4). Obviously $f$ satisfies the condition (2.10),

$$
\left(1^{2}, b^{\prime}\right)=\int_{0}^{1} 1 d t=1, \quad\left(1, a^{\prime}\right)=\int_{0}^{1} t d t=\frac{1}{2} .
$$

Hence by Theorem 2.4, we have

$$
\int_{C_{a, b}[0,1]}^{\mathrm{anf}_{\mathrm{q}}} F(x) d \mu(x)=\exp \left\{-\frac{i}{2 q}+i\left(\frac{i}{q}\right)^{1 / 2} \frac{1}{2}\right\} .
$$

Now we consider the right hand side of (3.4). Let $\Gamma\left(\lambda_{n}\right)$ be the function space integral on the right hand side of (3.4). By the same method as in the proof of 
Theorem 3.2, we have

$$
\begin{aligned}
\Gamma\left(\lambda_{n}\right)=K\left(1, \lambda_{n}\right)= & \lambda_{n}^{-n / 2} \exp \left\{-\frac{1}{2}\left(\psi_{n}^{2}, 1\right)+i\left(\psi_{n}, t\right)\right. \\
& \left.+\sum_{k=1}^{n}\left[-\frac{1}{2 \lambda_{n}} B_{k}\left(\phi_{k}, 1\right)_{a, b}^{2}+i \lambda_{n}^{-1 / 2} A_{k}\left(\phi_{k}, 1\right)_{a, b}\right]\right\},
\end{aligned}
$$

where

$$
\psi_{n}=1-\sum_{k=1}^{n}\left(\phi_{k}, 1\right)_{a, b} \phi_{k} .
$$

A careful look at the proof of Theorem 3.2 tells us that the convergences in (3.6), (3.7) and (3.8) are valid even if $a(t)$ does not satisfy the assumption in Theorem 3.2; that is,

$$
\left(\psi_{n}^{2}, 1\right) \rightarrow 0, \quad\left(\psi_{n}, t\right) \rightarrow 0, \quad \sum_{k=1}^{n} A_{k}\left(\phi_{k}, 1\right)_{a, b} \rightarrow(1, t)=\frac{1}{2}
$$

as $n \rightarrow \infty$. On the other hand,

$$
\begin{gathered}
B_{1}=\left(\phi_{1}^{2}, 1\right)=\int_{0}^{1} \frac{1}{1+t} d t=\ln 2 \\
\left(\phi_{1}, 1\right)_{a, b}=\int_{0}^{1} \frac{1}{\sqrt{1+t}}(1+t) d t=\frac{2}{3}(2 \sqrt{2}-1),
\end{gathered}
$$

and so

$$
B_{1}\left(\phi_{1}, 1\right)_{a, b}^{2}=\frac{4}{9}(9-4 \sqrt{2}) \ln 2 \approx 1.0299>1 .
$$

Since $B_{k} \geq 0$ for all $k=1,2, \ldots$, we have

$$
\sum_{k=1}^{n} B_{k}\left(\phi_{k}, 1\right)_{a, b}^{2} \geq B_{1}\left(\phi_{1}, 1\right)_{a, b}^{2}>1,
$$

and so the left hand side of the above inequality does not converge to 1 as $n \rightarrow \infty$. Hence

$$
\lim _{n \rightarrow \infty} \lambda_{n}^{n / 2} \Gamma\left(\lambda_{n}\right) \neq \exp \left\{-\frac{i}{2 q}+i\left(\frac{i}{q}\right)^{1 / 2} \frac{1}{2}\right\} .
$$

Comparing (3.13) and (3.14), we see that the relationship (3.4) is not valid in this case.

Theorem 3.5. Let $|a(t)|=c b(t)$ on $[0, T]$ for some constant $c \geq 0$. Let $\left\{\phi_{n}\right\}$ be a complete orthonormal set of functionals in $L_{a, b}^{2}[0, T]$. Let $\lambda \in \mathbb{C}_{+}$. Let $F \in$ $\mathcal{S}\left(L_{a, b}^{2}[0, T]\right)$ be given by $(2.4)$ and suppose that the associated measure $f$ satisfies condition (2.8). Then

$$
\begin{aligned}
& \int_{C_{a, b}[0, T]}^{\operatorname{an}_{\lambda}} F(x) d \mu(x) \\
= & \lim _{n \rightarrow \infty} \lambda^{n / 2} \int_{C_{a, b}[0, T]} \exp \left\{\frac{1-\lambda}{2} \sum_{k=1}^{n} \frac{\left\langle\phi_{k}, x\right\rangle^{2}}{B_{k}}+\left(\lambda^{1 / 2}-1\right) \sum_{k=1}^{n} \frac{A_{k}\left\langle\phi_{k}, x\right\rangle}{B_{k}}\right\} F(x) d \mu(x) .
\end{aligned}
$$


Proof. To prove this corollary, we modify the first part of the proof of Theorem 3.2 by replacing $\lambda_{n}$ by $\lambda$ whenever it occurs. Then we have

$$
\lim _{n \rightarrow \infty} \lambda^{n / 2} K(v, \lambda)=\exp \left\{-\frac{1}{2 \lambda}\left(v^{2}, b^{\prime}\right)+i \lambda^{-1 / 2}\left(v, a^{\prime}\right)\right\} .
$$

Since $f$ satisfies condition (2.8) we apply the dominated convergence theorem to obtain

$$
\lim _{n \rightarrow \infty} \lambda^{n / 2} \Gamma(\lambda)=\int_{L_{a, b}^{2}[0, T]} \exp \left\{-\frac{1}{2 \lambda}\left(v^{2}, b^{\prime}\right)+i \lambda^{-1 / 2}\left(v, a^{\prime}\right)\right\} d f(v) .
$$

By (2.9) in Theorem 2.4, the proof is completed.

Our main result, namely a change of scale formula for a function space integral on $C_{a, b}[0, T]$, now follows from Theorem 3.5.

Theorem 3.6. Let $|a(t)|=c b(t)$ on $[0, T]$ for some constant $c \geq 0$. Let $\left\{\phi_{n}\right\}$ be a complete orthonormal set of functionals in $L_{a, b}^{2}[0, T]$. Let $\rho>0$ and let $F \in$ $\mathcal{S}\left(L_{a, b}^{2}[0, T]\right)$ be given by $(2.4)$. Then

$$
\begin{aligned}
& \int_{C_{a, b}[0, T]} F(\rho x) d \mu(x) \\
= & \lim _{n \rightarrow \infty} \rho^{-n} \int_{C_{a, b}[0, T]} \exp \left\{\frac{\rho^{2}-1}{2 \rho^{2}} \sum_{k=1}^{n} \frac{\left\langle\phi_{k}, x\right\rangle^{2}}{B_{k}}+\left(\rho^{-1}-1\right) \sum_{k=1}^{n} \frac{A_{k}\left\langle\phi_{k}, x\right\rangle}{B_{k}}\right\} F(x) d \mu(x) .
\end{aligned}
$$

Proof. By letting $\lambda=\rho^{-2}$ in (3.15), we have equation (3.16). Note that since $\operatorname{Im}\left(\lambda^{-1 / 2}\right)=\operatorname{Im} \rho=0$, condition $(2.8)$ is satisfied for every $f$ in $M\left(L_{a, b}^{2}[0, T]\right)$.

Taking $a(t)=0$ and $b(t)=t$ in Theorem 3.6, we have the following change of scale formula for Wiener integrals on classical Wiener space.

Corollary 3.7 (Theorem 2 of [4]). Let $\left\{\phi_{n}\right\}$ be a complete orthonormal set of functionals in $L^{2}[0, T]$. Let $\rho>0$ and let $F \in \mathcal{S}$ be given by (3.11). Then

$$
\int_{C_{0}[0, T]} F(\rho x) d m_{w}(x)=\lim _{n \rightarrow \infty} \rho^{-n} \int_{C_{0}[0, T]} \exp \left\{\frac{\rho^{2}-1}{2 \rho^{2}} \sum_{k=1}^{n}\left\langle\phi_{k}, x\right\rangle^{2}\right\} F(x) d m_{w}(x) .
$$

\section{REFERENCES}

1. R.H. Cameron, The translation pathology of Wiener space, Duke Math. J. 21 (1954), 623-628. MR0065033 (16:375b)

2. R.H. Cameron and W.T. Martin, The behavior of measure and measurability under change of scale in Wiener space, Bull. Amer. Math. Soc. 53 (1947), 130-137. MR0019259 (8:392a)

3. R.H. Cameron and D.A. Storvick, Some Banach algebras of analytic Feynman integrable functionals, in Analytic Functions (Kozubnik, 1979), Lecture Notes in Math. 798, SpringerVerlag (1980), 18-67. MR.577446 (83f:46059)

4. Change of scale formulas for Wiener integral, Supplemento ai Rendiconti del Circolo Matematico di Palermo, Serie II-Numero 17 (1987), 105-115. MR950411 (90d:28017)

5. __ Relationships between the Wiener integral and the analytic Feynman integral, Supplemento ai Rendiconti del Circolo Matematico di Palermo, Serie II-Numero 17 (1987), 117-133. MR950412 (90b:28014)

6. S.J. Chang, J.G. Choi and D. Skoug, Integration by parts formulas involving generalized Fourier-Feynman transforms on function space, Trans. Amer. Math. Soc. 355 (2003), 29252948. MR.1975406 (2004c:60229) 
7. _ Parts formulas involving conditional generalized Feynman integrals and conditional generalized Fourier-Feynman transforms on function space, Integral Transform. Spec. Funct. 15 (2004), 491-512. MR2094565 (2005i:60154)

8. S.J. Chang and I.Y. Lee, A Fubini theorem for generalized analytic Feynman integrals and Fourier-Feynman transforms on function space, Bull. Korean Math. Soc. 40 (2003), 437-456. MR.1996853 (2004g:28018)

9. S.J. Chang and D. Skoug, Generalized Fourier-Feynman transforms and a first variation on function space, Integral Transform. Spec. Funct. 14 (2003), 375-393. MR2005996 (2004h:81098)

10. G.W. Johnson and D.L. Skoug, Scale-invariant measurability in Wiener space, Pacific J. Math. 83 (1979), 157-176. MR555044 (81b:28016)

11. J. Yeh, Stochastic processes and the Wiener integral, Marcel Dekker, Inc., New York, 1973. MR0474528 (57:14166)

12. I. Yoo and D. Skoug, A change of scale formula for Wiener integrals on abstract Wiener spaces, Internat. J. Math. Math. Sci. 17 (1994), 239-248. MR.1261069 (95c:28013)

13. _ A change of scale formula for Wiener integrals on abstract Wiener spaces II, J. Korean Math. Soc. 31 (1994), 115-129. MR.1269456 (95c:28014)

14. I. Yoo, T.S. Song and B.S. Kim, A change of scale formula for Wiener integrals of unbounded functions II, Commun. Korean Math. Soc. 21 (2006), 117-133. MR2199306 (2007c:28012)

15. I. Yoo, T.S. Song, B.S. Kim and K.S. Chang, A change of scale formula for Wiener integrals of unbounded functions, Rocky Mountain J. Math. 34 (2004), 371-389. MR2061137 (2005a:28027)

Department of Mathematics, Yonsei University, Wonju 220-710, Republic of Korea

E-mail address: iyoo@yonsei.ac.kr

Department of Mathematics, Daejin University, Pocheon 487-711, Republic of Korea

E-mail address: bjkim@daejin.ac.kr

School of Liberal Arts, Seoul National University of Science and Technology, Seoul 139-743, Republic of Korea

E-mail address: mathkbs@seoultech.ac.kr 